\title{
Using a Post-Intubation Checklist and Time Out to Expedite Mechanical Ventilation Monitoring: Observational Study of a Quality Improvement Intervention
}

\author{
Ryan A McConnell MD, Meeta Prasad Kerlin MD MSCE, William D Schweickert MD, \\ Faraz Ahmad MD, Mitesh S Patel MD MBA MSc, and Barry D Fuchs MD
}

\begin{abstract}
BACKGROUND: Delayed mechanical ventilation monitoring may impede recognition of lifethreatening acidemia. Coordination of multidisciplinary processes can be improved by using a checklist and time-out procedure. The study objective was to evaluate process-related outcomes after implementation of a post-intubation checklist and time out. METHODS: An observational study of a 24-bed medical ICU in Philadelphia, Pennsylvania, was conducted from January to December 2011. A random sample of mechanically ventilated adults was selected from the preintervention $(n=80)$ and post-intervention $(n=144)$ periods. The primary outcome was the proportion of subjects with an arterial blood gas (ABG) result within $60 \mathrm{~min}$ of mechanical ventilation initiation. Secondary outcomes included rates of respiratory acidosis, moderate-severe acidemia ( $\mathrm{pH}<7.25)$, checklist initiation, and project sustainability. Chi-square analysis was used to evaluate differences in outcomes between time periods. RESULTS: After the intervention, the proportion of subjects with an $\mathrm{ABG}$ result within $60 \mathrm{~min}$ increased $(56 \% \mathrm{vs} 37 \%, P=.01)$, and time to $\mathrm{ABG}$ result improved (58 $\mathrm{min}$ vs $79 \mathrm{~min}, P=\mathbf{. 0 0 4}$ ). Adjusting for illness severity, the proportion with an ABG result within $60 \mathrm{~min}$ remained significantly higher in the post-intervention period (odds ratio $2.42,95 \% \mathrm{CI} 1.25-4.68, P=.009$ ). Checklist adherence was higher with ICU intubations than for intubations performed outside the ICU $(71 \%$ vs $27 \%$ checklist initiation rate, $P<.001)$. Transfer from referring institutions $(23 \%$ checklist initiation rate, $P=.006)$ negatively impacted checklist use. Implementation challenges included frequent stakeholder turnover, undefined process ownership, and lack of real-time performance feedback. CONCLUSIONS: A post-intubation checklist and time out improved the timeliness of mechanical ventilation monitoring through more rapid assessment of arterial blood gases. Implementing this peri-intubation procedure may reduce the risks associated with transitioning to full mechanical ventilatory support. Optimal implementation necessitates strategies to surmount organizational and behavioral barriers to change. Key words: blood gas analysis; acidosis; artificial respiration; mechanical ventilators; checklist; quality improvement. [Respir Care 2016;61(7):902-912. () 2016 Daedalus Enterprises]
\end{abstract}

\section{Introduction}

Mechanical ventilation is one of the most common intensive care interventions, ${ }^{1}$ with $>790,000$ United

Dr McConnell is affiliated with the Division of Gastroenterology, Department of Medicine, University of California, San Francisco, San Francisco, California. Drs Prasad Kerlin, Schweickert, and Fuchs are affiliated with the Division of Pulmonary, Allergy, and Critical Care, Department of Medicine, University of Pennsylvania, Philadelphia, Pennsylvania. Dr Ahmad is affiliated with the Division of Cardiology,
States hospitalizations involving this life-sustaining therapy annually. ${ }^{2}$ The transition to mechanical ventilation represents a vulnerable time for patients, because vital physiologic processes are now controlled by an external

\footnotetext{
Department of Medicine, Northwestern University, Chicago, Illinois. Dr Patel is affiliated with the Department of Medicine, University of Pennsylvania, and Philadelphia Veterans Affairs Medical Center, Philadelphia, Pennsylvania.
}

The authors have disclosed no conflicts of interest. 
source. Insufficient ventilator settings can lead to acid/base disturbances when anesthesia or sedation prevents or attenuates the patient's compensatory respiratory response. The patient is most vulnerable in the period immediately following mechanical ventilation initiation, a time when the patient's condition is often rapidly deteriorating and clinicians can only estimate the ventilator settings most appropriate for the patient's physiology. Inadequate ventilation is especially dangerous for patients with preexisting metabolic acidosis, a condition commonly present at the time of ICU admission. ${ }^{3}$

\section{See the Related Editorial on Page 996}

A blood gas analysis must be performed to assess the adequacy of mechanical ventilator settings. Arterial blood gas (ABG) is preferred over venous blood gas in acutely ill patients who commonly have cardiovascular dysfunction, because arterial and venous measurements correlate poorly in this population. ${ }^{4-6}$ The $\mathrm{P}_{\mathrm{CO}_{2}}$ in arterial blood $\left(\mathrm{P}_{\mathrm{aCO}}\right)$ reaches a new steady state approximately 10-20 min following a change in minute ventilation, ${ }^{7,8}$ whereas the $\mathrm{P}_{\mathrm{aO}_{2}}$ attains equilibrium even faster. ${ }^{9,10}$ Despite the importance of timely ABG measurement, there are no guidelines or published standards that promote this practice. The peri-intubation period is frequently complicated by competing priorities to stabilize acutely ill patients, which may delay ABG collection. In addition, the peri-intubation care process involves multiple stakeholders (eg, physician, nurse, respiratory therapist). Ineffective coordination of care and role ambiguity can lead to significant delays in ABG monitoring, thereby putting the patient at risk. ${ }^{11,12}$ Performance audits revealed wide variability in ABG timing and a high rate of post-intubation acidemia across multiple ICUs at our institution.

Recent evidence suggests that coordination of multidisciplinary processes can be improved by using checklists and time out procedures. ${ }^{13-16}$ These methods take an unstructured process and apply a standardized protocol and deliberate pause to encourage communication and reduce variations in care. During this process, team members from

Dr McConnell presented preliminary data as a poster at the American College of Physicians National Meeting, held April 19-21, 2012, in New Orleans, Louisiana.

Supplementary material related to this paper is available at http:// www.rcjournal.com.

Correspondence: Ryan A McConnell MD, University of California, San Francisco, Division of Gastroenterology, 513 Parnassus Avenue, S-357, San Francisco, CA 94143. E-mail: ryan.mcconnell@ucsf.edu.

DOI: $10.4187 /$ respcare.04191

\section{QUICK LOOK}

\section{Current knowledge}

The initiation of mechanical ventilation is a vulnerable event for critically ill patients, because insufficient estimated ventilator settings can rapidly lead to life-threatening acid/base disturbances. Arterial blood gas measurement is a reliable tool to assess the adequacy of ventilation, although there are no established guidelines promoting timely blood gas acquisition. Checklists and procedural time outs improve the coordination of multidisciplinary processes, and critical care checklists have been successfully employed to reduce health care-associated infections.

\section{What this paper contributes to our knowledge}

A multidisciplinary post-intubation checklist and time out were developed for use in the medical ICU. After checklist implementation, a greater proportion of subjects received an arterial blood gas within $1 \mathrm{~h}$ of initiating mechanical ventilation. Checklist adherence was associated with faster blood gases in a dose-dependent manner. Respiratory acidosis was frequently detected.

all disciplines review the plan of care in an effort to better coordinate responsibilities, ensure timeliness of action, and reaffirm agreement upon the proposed plan.

The use of a checklist has been demonstrated to effectively improve the quality of intensive care by reducing catheter-related bloodstream infections ${ }^{17}$ and ventilatorassociated pneumonias. ${ }^{18}$ Checklists also improve team performance in crisis situations, in which even basic tasks can be overlooked. ${ }^{19}$ However, we are unaware of checklist use to coordinate the complex care processes that occur routinely during the post-intubation period, including management of post-intubation analgesia and sedation; obtaining a chest radiograph, sputum culture, and ABG; and ventilator setting adjustment. The objective of this study was to evaluate the process-related outcomes after implementation of a post-intubation checklist and time out during initiation of mechanical ventilation.

\section{Methods}

\section{Study Design}

We conducted a retrospective observational study of a 24-bed medical ICU (MICU) in Philadelphia, Pennsylvania, from January to December 2011. A post-intubation checklist and time out were implemented on May 2, 2011. A random sample of mechanically ventilated adults was selected for evaluation from the pre-intervention $(n=80)$ 
and post-intervention $(n=144)$ periods. This study was approved by the institutional review board of the University of Pennsylvania. A waiver of informed consent was approved because this study posed minimal risk to patient confidentiality, and retrospective evaluation would otherwise have been infeasible.

\section{Setting and Subjects}

The intervention was conducted in the MICU at the Hospital of the University of Pennsylvania, an urban, universityaffiliated, tertiary care referral hospital. The MICU is staffed by 2 teams, each consisting of one pulmonary/critical care attending physician, one critical care fellow, 4 residents, 2 interns, one advanced practice provider, and one pharmacist. Attending physicians, fellows, and house staff spend 2-4 consecutive weeks on service. Two to three respiratory therapists are integrated across both teams. The nurse/patient ratio is $1: 1$ or $1: 2$, depending on the MICU occupancy and acuity of individual patients. Blood gas analysis is performed in a central laboratory offsite.

The sample comprised patients initiated on mechanical ventilation in the MICU between January 5 and December 25,2011 . The start date was selected because it was the day after a departmental morbidity and mortality review focused attention on mechanical ventilation monitoring, and we believed that clinical practice would change accordingly. Therefore, choosing a start date after the departmental review was intended to mitigate this potential source of confounding. We selected a 1-y study eligibility period because of seasonal variation in house staff experience and in case mix. To be eligible for inclusion, subjects were required to have at least one $\mathrm{ABG}$ measurement after initiating mechanical ventilation, and data on ventilator settings must have been available for review. Patients monitored exclusively by venous blood gas were excluded, because arterial and venous measurements correlate poorly in certain clinical conditions, ${ }^{4-6}$ and our local practice restricts venous blood gas monitoring to ventilator weaning in stable patients. To reduce the likelihood that analyses were affected by correlations within repeated measures, we included only the first mechanical ventilation episode for a subject during a single hospitalization.

Subjects were identified retrospectively via mechanical ventilation registry records. To select a random sample of subjects from the pre- and post-intervention periods, each patient was assigned a number through a computerized random number generator. Subjects with numbers 1-224 were selected for chart review.

\section{Intervention}

The multidisciplinary unit-based clinical leadership committee, consisting of physician and nursing leadership, resident physicians, and a quality improvement specialist, developed the Post-Intubation Time Out process and checklist using PDSA (plan-do-study-act) methodology. ${ }^{20}$ This intervention was designed to guide and expedite approximately 20 routine patient care tasks at the initiation of mechanical ventilation. The checklist organizes workflow and delegates responsibility for each task to the physician, nurse, or respiratory therapist (Fig. 1). At the time the patient is connected to the MICU ventilator (time zero), the physician, nurse, and respiratory therapist huddle to perform a time out and discuss the ventilation strategy. Initial checklist tasks include order entry, documentation of initial ventilator settings and lung mechanics, and assignment of an individual to collect the ABG. Personnel are encouraged to set mobile telephone alarms for 15 and 60 min to prompt ABG collection and checklist completion, respectively. Within $60 \mathrm{~min}$, these same 3 personnel meet again to complete the follow-up checklist, confirming completion of the assigned tasks and documenting ventilator adjustments in response to the $\mathrm{ABG}$ result.

This process was intended for all patients at the onset of mechanical ventilation in the MICU, including those intubated elsewhere who arrived on a ventilator. We opted to include even those patients for whom mechanical ventilation was initiated before MICU admission because clinical conditions may change significantly during intra- and interhospital patient transport, ${ }^{21-24}$ particularly if sedatives or paralytics are administered, and we believe that it is prudent to reassess ventilation adequacy with an $\mathrm{ABG}$ upon MICU arrival. Furthermore, in our hospital, patients intubated on wards other than an ICU often are bag-ventilated until ICU arrival, at which time they are connected to the ventilator (time zero).

Project rationale and instructions were disseminated in multiple educational sessions for all stakeholders. House staff $(n=165)$ attended 1-h case-based ventilator physiology and patient safety conferences highlighting the project. They also received explanatory emails before each MICU rotation and a project overview in monthly MICU orientations. Project leaders educated nurses $(n=85)$ and respiratory therapists $(n=90)$ at daily shift huddles and monthly unitwide meetings. The checklist underwent 18 iterative revisions in response to stakeholder feedback and pilot testing. All revisions were finalized prior to the implementation date.

The checklist intervention was implemented on May 2, 2011, as the new standard of care for all mechanically ventilated MICU patients. On June 7, 2011, a mechanical ventilation order set linked to the checklist was implemented within the electronic health record to assist providers with bundled order entry, including orders for ABG, 


\section{Post Intubation TIME OUT}

DATE EXACT TIME OF INTUBATION MEMBERS PRESENT

$\begin{array}{ll}\square \text { Nurse } & \square \text { ICU Fellow } \\ \square \text { Respiratory Therapist } & \square \text { ICU Attending } \\ \square \text { Primary Resident } & \square \text { Anesthesia }\end{array}$

\begin{tabular}{ll|}
\hline Difficult $\square$ No & $\square$ Yes \\
Airway: & $\square$ Wrist band applied \\
& $\square$ Documentation completed
\end{tabular}

Time Out Checklist

Complete immediately upon intubation or ICU arrival

MD
$\square$ Lungs ausculated, breath sounds confirmed
$\square$ ABG ordered
$\square$ CXR ordered
$\square$ Sputum culture ordered (if indicated)
$\square$ Sedation ordered
$\square$ Mechanical Ventilation ordered
$\square$ Low Stretch Protocol ordered
(if bilateral opacities on pre-intubation CXR)

\section{RN}

$\square$ Record blood pressure:

Shock? $\square$ No $\square$ Yes $\rightarrow$ start IVF/pressors

RT
breaths
$\square$ Initial vent settings at time of intubation:
$\mathrm{CO}_{2}$ color change confirmed after 6
Mechanics:
Resistance
$\square$ I will wean $\mathrm{F}_{1 \mathrm{O}_{2}}$ as per vent liberation protocol.

ALL PARTIES

Person to collect ABG 15 min. post intubation:

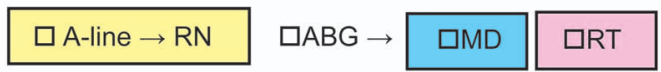

Name of collector:
CURRENT TIME

\section{CIRCUMSTANCE (Check all that apply) \\ $\square$ Elective Location of Intubation: \\ $\square$ Anesthesia STAT $\square$ HUP MICU $\square$ PPMC \\ $\square$ Code Call $\quad \square$ HUP CCU MICU \\ $\square$ Reintubation $\quad \square$ Floor $\quad \square$ Other \\ $\square$ Reintubation $\quad \square$ ED \\ post $\square \mathrm{OSH}$ \\ self-extubation $\quad \square \mathrm{OR}$}

\section{Follow-Up Checklist}

Complete within 1 hour of time out.

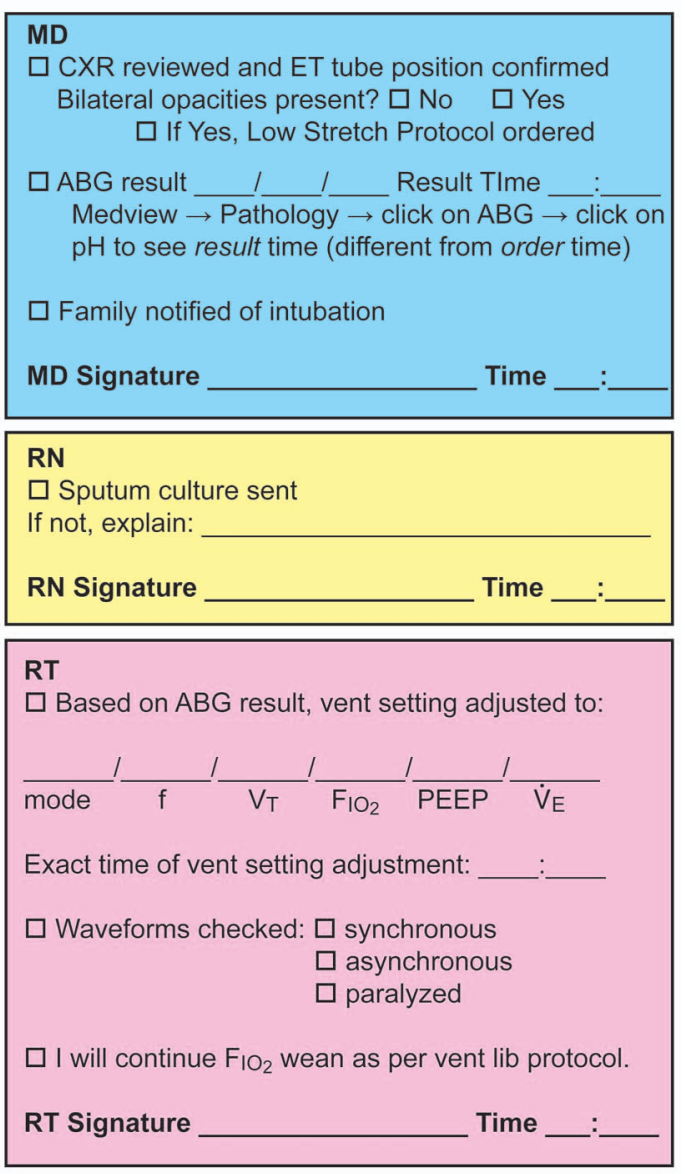

ALL PARTIES: Remember to re-check ABG and $V_{E}$ when patient spontaneously breathing.

\section{Comments:}

After completing of follow-up by all parties, please place on the RT Clipboard.

Fig. 1. Post-Intubation Time Out checklist. $M D=$ physician; $R N=$ nurse; $A B G=$ arterial blood gas; $C X R=$ chest radiograph; $R T=$ respiratory therapist; ET = endotracheal.

chest $\mathrm{x}$-ray, sputum culture, analgesia, sedation, and ventilator settings. In August 2011, a sustainability intervention was implemented whereby blank checklists were at- tached to every mechanical ventilator on stand-by, providing a visual cue to prompt checklist use at the initiation of mechanical ventilation. 


\section{Post-Intubation Checklist and Time Out to Expedite Mechanical Ventilation Monitoring}

\section{Data}

A single non-blinded reviewer (RAM) extracted data from charts, laboratory results, and radiology reports using a standardized data collection form. Ventilator flow sheets completed by respiratory therapists provided the time of initiation of mechanical ventilation in the MICU (time zero), initial ventilator settings, and time-stamped ventilator setting changes. The first ABG result and chest $\mathrm{x}$-ray report occurring after initiation of mechanical ventilation in the MICU were recorded. In rare instances when a venous blood gas was obtained before an $\mathrm{ABG}$, only the $\mathrm{ABG}$ result was recorded. Demographic information, firstday Acute Physiology and Chronic Health Evaluation (APACHE) III scores, ${ }^{25}$ and discharge disposition were obtained from a clinical registry.

\section{Main Outcome Measures}

The primary outcome measure was the proportion of subjects with an ABG result in 60 min or less from the time of MICU mechanical ventilation initiation. This 1-h cutoff is consistent with prior studies, whose aim was to establish specific criteria for ordering ABGs. ${ }^{26,27}$ In addition, this seemed like a reasonable threshold, given the time it takes for $\mathrm{P}_{\mathrm{aCO}_{2}}$ to equilibrate following a ventilation change $\mathrm{e}^{7,8}$ and anticipated variability in specimen transport and processing time using the central laboratory offsite. Secondary outcome measures included time from initiation of mechanical ventilation to $\mathrm{ABG}$ result, frequency of moderate-severe acidemia on initial post-intubation $A B G$ (defined as $\mathrm{pH}<7.25$ ), and frequency of respiratory acidosis.

We also measured 2 implementation outcomes in secondary analyses: fidelity and sustainability. Fidelity was measured as the proportion of subjects in the post-intervention period for which the checklist was initiated. Sustainability was measured as the proportion of subjects for which the checklist was used on a monthly basis over the duration of the study.

We performed a pre-specified subgroup analysis on subjects intubated in the MICU because patients intubated before MICU transfer have often already undergone initial $\mathrm{ABG}$ assessment and ventilator adjustment; therefore, this pre-ICU care might alter the manner in which these patients are treated on arrival. For this subgroup, we also assessed an additional secondary outcome measure of time to achieve an $\mathrm{F}_{\mathrm{IO}_{2}}$ of $<0.7$. $\mathrm{F}_{\mathrm{IO}_{2}}$ weaning was an MICU expectation to avoid unnecessary exposure to toxic levels of oxygen, which may cause lung injury. ${ }^{28}$

\section{Statistical Analysis}

The Post-Intubation Time Out intervention was implemented on May 2, 2011. The exposure variable was de- fined according to the date of initiation of mechanical ventilation relative to the implementation date. Subjects initiated before implementation (pre-intervention) were considered unexposed, and subjects initiated on or after the implementation date (post-intervention) were considered exposed.

Data were summarized using standard descriptive statistics. The primary comparison was made on an intentionto-treat basis. The chi-square test and Wilcoxon rank-sum test were used as appropriate for univariate tests of association. Pearson $r$ was used to assess the correlation between checklist completeness and time to ABG. For subjects in the post-intervention period, univariate tests were performed to determine which characteristics were associated with the use of the checklist.

A minimum sample size of 49 subjects in each exposure group was required to achieve $80 \%$ power with a 2 -sided $\alpha$ of 0.05 to detect a doubling of the proportion of subjects with an initial ABG result within $60 \mathrm{~min}$, assuming a baseline proportion of $27 \%$ (based on historical data from the study ICU before the departmental morbidity and mortality review). We anticipated a $50 \%$ checklist initiation rate and also that $15 \%$ of subjects might be excluded based on pre-specified exclusion criteria; therefore, we randomly selected 224 subjects to ensure an adequate sample size.

All tests were 2-tailed with a $P$ value of $<.05$ considered significant. Analyses were performed by an independent statistician using SAS 9.3 (SAS Institute, Cary, North Carolina).

\section{Results}

Among the 586 patients who underwent mechanical ventilation during the study eligibility period, 214 (37\%) were in the pre-intervention period and $372(63 \%)$ were in the post-intervention period. We evaluated 224 randomly selected subjects: 80 from the pre-intervention period and 144 from the post-intervention period (Fig. 2). Thirty-six subjects were excluded. The excluded subjects included 13 monitored exclusively by venous blood gas ( 7 had tracheostomies and were undergoing ventilator weaning, 2 had hypercarbic respiratory failure, one was intubated for $<12 \mathrm{~h}$, and one was intubated during cardiopulmonary resuscitation and subsequently died), 10 with ventilator documents missing from the archived medical record, and 9 for whom no blood gas was performed (3 were tolerating tracheostomy collar and required only limited nocturnal ventilation, 2 were admitted with non-pulmonary pathology and required the MICU for chronic nocturnal ventilation, one required brief intubation for upper endoscopy, one was intubated for alcohol intoxication and rapidly selfextubated, and one chronic ventilator facility resident was admitted with a displaced gastrostomy tube). The final analysis included 188 subjects: $70(37 \%)$ from the pre- 


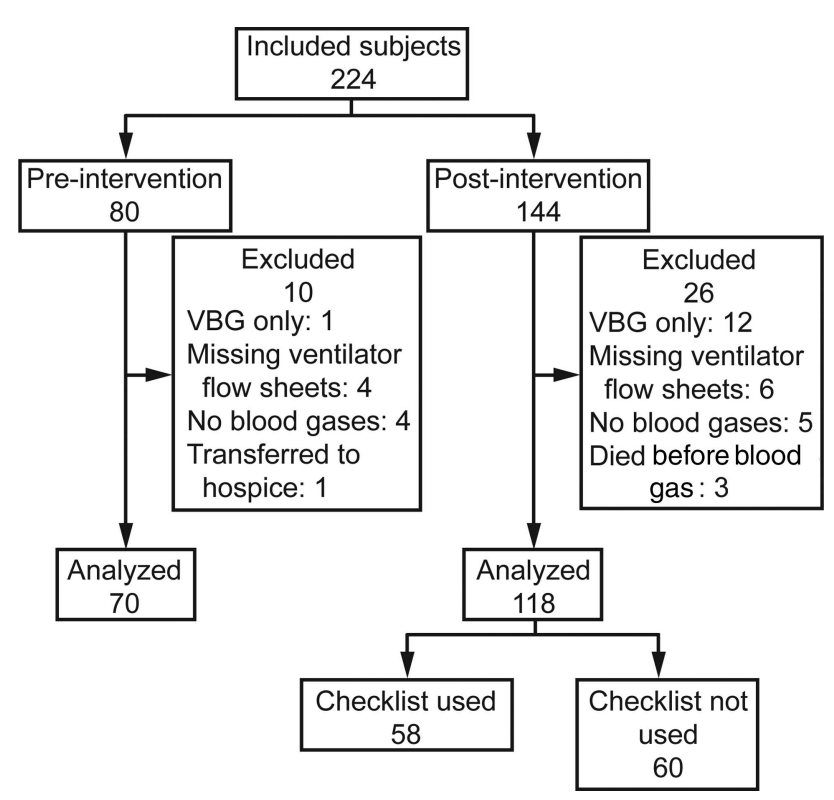

Fig. 2. Flow chart. VBG = venous blood gas.

intervention period and $118(63 \%)$ from the post-intervention period.

Subject demographics were similar across the pre-intervention and post-intervention groups, with the exception that the pre-intervention group had a slightly higher median APACHE III score and a nonsignificant trend toward more admissions from the emergency department (Table 1). Seasonal variation may explain this distribution, with the pre-intervention period spanning most of the winter months. Just over half of the subjects $(n=97,52 \%)$ were already intubated before MICU arrival. Mortality did not differ between the groups.

The proportion of subjects with an ABG result within 60 min was significantly greater in the post-intervention group than in the pre-intervention group $(n=66 / 118$ [56\%] vs $n=26 / 70$ [37\%], univariate $P=.01$ ) (Table 2). Repeating the analysis to include the 13 subjects monitored exclusively by venous blood gas did not alter the magnitude or statistical significance of the findings. After adjustment for severity of illness, subjects in the post-intervention period had significantly higher odds of having an ABG result within 60 min (odds ratio 2.42, 95\% CI 1.25$4.68, P=.009$ ) when compared with the pre-intervention period. The median time to $\mathrm{ABG}$ was significantly lower in the post-intervention group (58 vs $79 \mathrm{~min}, P=.004$ ). Respiratory acidosis (range $41-56 \%$ ) and moderate-severe acidemia (range 27-35\%) occurred frequently in both groups but did not differ significantly between the groups. Similar trends were observed for the subgroup of subjects intubated in the MICU, with an even higher prevalence of respiratory acidosis and acidemia (Table 2). The time to attain $\mathrm{F}_{\mathrm{IO}_{2}}<0.7$ was not significantly different between the pre- and post-intervention groups $(P=.77)$.
Checklists were initiated for 58 subjects (49\%) during the post-intervention period. Checklist initiation was significantly greater among subjects intubated in the MICU than for subjects intubated before MICU arrival $(n=42 / 59$ [71\%] vs $n=16 / 59$ [27\%], $P<.001)$ and among those with initiation of mechanical ventilation overnight compared with during the daytime $(n=35 / 59$ [59\%] vs $n=23 / 59$ [39\%], $P=.02$ ). Lower rates of checklist initiation were found among subjects transferred from a referring institution $(n=6 / 26$ [23\%], $P=.006)$ and for those receiving a pressure-limited ventilator mode $(n=4 / 18$ [22\%], $P=.01)$. Severity of illness did not significantly affect checklist use (median APACHE III score 97 for subjects with a checklist initiated vs 92 for subjects without a checklist initiated, $P=.42$ ).

The degree of checklist completeness varied, with over half of initiated checklists completed only partially. Protocol adherence (ie, completing the checklist) was strongly associated with timely ABG results (Fig. 3). There was a significant inverse correlation between number of checklist items completed and time to $\operatorname{ABG}(P=.01)$. A designated individual to collect the ABG was assigned on 46 of the 58 checklists (79\%). When a specific individual was designated, $61 \%$ of $\mathrm{ABG}$ results were obtained within 60 min, compared with $50 \%$ when no one was designated $(P=.50)$.

Figure 4 depicts the proportion of subjects with an $A B G$ result within $60 \mathrm{~min}$ and the checklist initiation rate according to study month. There was an increase in the proportion of subjects achieving the primary outcome following the departmental morbidity and mortality review. There was a marked and abrupt but transient increase in timely ABGs coincident with the May implementation of the Post-Intubation Time Out. Performance then returned to baseline levels and did not improve with the June implementation of the electronic order set. Performance reached its nadir in July, a transition month in the United States academic calendar marked by an influx of new house staff unfamiliar with the project. In August, we began attaching blank checklists to every mechanical ventilator on stand-by. Following this sustainability intervention, there was a consistent improvement in the proportion of subjects achieving the primary outcome for the remainder of the study.

\section{Discussion}

We designed, implemented, and sustained a multidisciplinary checklist and communication tool, the use of which was associated with a $>50 \%$ increase in the proportion of subjects receiving an $\mathrm{ABG}$ result within $60 \mathrm{~min}$ of initiating mechanical ventilation. However, sustained performance improvement was not achieved until the final 4 months of the study, when $>65 \%$ of subjects met the 


\section{Post-Intubation Checklist and Time Out to Expedite Mechanical Ventilation Monitoring}

Table 1. Characteristics of Study Subjects

\begin{tabular}{|c|c|c|c|}
\hline & Pre-Intervention $(n=70)$ & Post-Intervention $(n=118)$ & $P$ \\
\hline Female sex, $n(\%)$ & $32(46)$ & $56(47)$ & .82 \\
\hline Age, median (IQR) y & $62(48-69)$ & $59(48-67)$ & .74 \\
\hline Race, $n(\%)$ & & & .38 \\
\hline Caucasian & $35(50)$ & $69(59)$ & \\
\hline African-American & $29(41)$ & $37(31)$ & \\
\hline Other & $6(9)$ & $12(10)$ & \\
\hline Body mass index, median (IQR) $\mathrm{kg} / \mathrm{m}^{2}$ & $27.4(24.8-30.7)$ & $28(24-33.5)$ & .68 \\
\hline APACHE III score, median (IQR) & $110(91-134)$ & $95(75-122)$ & .02 \\
\hline Source of MICU admission, $n(\%)$ & & & .07 \\
\hline Emergency department & $28(40)$ & $26(22)$ & \\
\hline Medical/surgical floor & $27(39)$ & $55(47)$ & \\
\hline Other ICU or operating room & $4(6)$ & $11(9)$ & \\
\hline Referring institution & $11(16)$ & $26(22)$ & \\
\hline Location of Intubation, $n(\%)$ & & & .57 \\
\hline $\mathrm{MICU}$ & $32(46)$ & $59(50)$ & \\
\hline Non-MICU & $38(54)$ & $59(50)$ & \\
\hline Time of mechanical ventilation initiation in MICU, $n(\%)$ & & & .30 \\
\hline Rounds (0700-1200) & $9(13)$ & $20(17)$ & \\
\hline Afternoon (1201-1900) & $31(44)$ & $39(33)$ & \\
\hline Overnight (1901-0659) & $30(43)$ & $59(50)$ & \\
\hline Initial ventilator mode, $n(\%)$ & & & .18 \\
\hline Volume controlled & $64(91)$ & $100(85)$ & \\
\hline Pressure controlled & $6(9)$ & $18(15)$ & \\
\hline Initial set breathing frequency, median (IQR) breaths/min & $18(14-24)$ & $20(16-22)$ & .96 \\
\hline Initial tidal volume, median (IQR) $\mathrm{mL}$ & $500(425-550)$ & $450(400-550)$ & .24 \\
\hline Initial $\mathrm{F}_{\mathrm{IO}_{2}}$, median $(\mathrm{IQR})$ & $1(1-1)$ & $1(0.6-1)$ & .01 \\
\hline Initial PEEP, median (IQR) $\mathrm{cm} \mathrm{H}_{2} \mathrm{O}$ & $5(5-7.5)$ & $5(5-7.5)$ & .11 \\
\hline Initial set minute ventilation, median (IQR) $\mathrm{L} / \mathrm{min}$ & $10(8.3-12.4)$ & $9.8(7.9-11.5)$ & .49 \\
\hline Bilateral infiltrates on post-intubation CXR, $n(\%)$ & $35(50)$ & $54(46)$ & .57 \\
\hline Hospital discharge disposition, $n(\%)$ & & & .39 \\
\hline Death or hospice & $32(46)$ & $63(53)$ & \\
\hline Other medical facility & $25(36)$ & $31(26)$ & \\
\hline Home & $13(19)$ & $24(20)$ & \\
\hline \multicolumn{4}{|l|}{$\begin{array}{l}\text { IQR }=\text { interquartile range } \\
\text { APACHE }=\text { Acute Physiology and Chronic Health Evaluation } \\
\text { MICU = medical intensive care unit } \\
\text { CXR }=\text { chest radiograph }\end{array}$} \\
\hline
\end{tabular}

primary outcome. This delay may reflect a learning curve as personnel gained familiarity with the intervention. Checklist-driven behavioral changes often take time. For example, mortality reduction required a year to achieve in one study of a surgical safety checklist. ${ }^{30}$

Despite the demonstrated dose-response relationship between checklist completeness and the timeliness of ABG results, overall adherence to the checklist was only fair, at $49 \%$. Although comparable adherence rates have been reported with other checklist-based interventions, ${ }^{31-34}$ we were surprised by the dichotomous nature of checklist use. Whereas the majority of subjects intubated in the MICU had checklists initiated (71\%), adherence was much lower for subjects intubated outside of the MICU (27\%). This finding was especially pronounced among subjects admit- ted directly from referring institutions or long-term acute care hospitals on ventilators. Checklist underutilization may result from a perception that patients already receiving mechanical ventilation are more stable. Respiratory acidosis and moderate-severe acidemia were less prevalent in these subjects (36 and 23\% of non-MICU intubations vs 66 and $42 \%$ of MICU intubations, respectively), suggesting a certain degree of pre-MICU stabilization. However, clinical conditions may change significantly during intraand interhospital patient transport, ${ }^{21-24}$ and it is prudent to reassess ventilation adequacy with an ABG upon MICU arrival. Low checklist adherence was also observed for subjects receiving spontaneous modes of ventilation, perhaps because they too were perceived as more stable. The rationale for performing a time out in these instances may 
Table 2. Primary and Secondary Clinical Outcomes

\begin{tabular}{|c|c|c|c|}
\hline All Subjects & Pre-Intervention $(n=70)$ & Post-Intervention $(n=118)$ & $P$ (univariate) \\
\hline $\mathrm{ABG}$ result in $\leq 60 \mathrm{~min}, n(\%)$ & $26(37)$ & $66(56)$ & .01 \\
\hline Time to ABG, median (IQR) min & $79(44-117)$ & $58(35-95)$ & .004 \\
\hline Respiratory acidosis, $n(\%)^{*}$ & $29(41)$ & $66(56)$ & .054 \\
\hline $\mathrm{ABG} \mathrm{pH}<7.25$ & $19(27)$ & $41(35)$ & .28 \\
\hline Subjects Intubated in MICU & Pre-Intervention $(n=32)$ & Post-Intervention $(n=59)$ & $P$ (univariate) \\
\hline $\mathrm{ABG}$ result in $\leq 60 \mathrm{~min}$ & $14(44)$ & $34(58)$ & .21 \\
\hline Time to ABG, median (IQR) min & $74(45-106)$ & $57(37-72)$ & .040 \\
\hline Respiratory acidosis, $n(\%)^{*}$ & $17(53)$ & $43(73)$ & .058 \\
\hline $\mathrm{ABG} \mathrm{pH}<7.25, n(\%)$ & $12(38)$ & $26(44)$ & .54 \\
\hline Time to $\mathrm{F}_{\mathrm{IO}_{2}}<0.7$, median (IQR) $\mathrm{h}$ & $2.8(1.5-7)$ & $3.5(0.5-10.9)$ & .77 \\
\hline $\begin{array}{l}\mathrm{F}_{\mathrm{IO}_{2}}<0.7 \text { at initiation of } \\
\text { mechanical ventilation, } n(\%)\end{array}$ & $3(9)$ & $14(24)$ & .16 \\
\hline $\mathrm{F}_{\mathrm{IO}_{2}}<0.7$ never achieved, $n(\%)$ & $1(3)$ & $1(2)$ & $>.99$ \\
\hline $\begin{array}{l}{ }^{*} \text { Calculated using the Winters formula. } 29 \\
\text { ABG }=\text { arterial blood gas } \\
\text { IQR }=\text { interquartile range } \\
\text { MICU = medical intensive care unit }\end{array}$ & & & \\
\hline
\end{tabular}

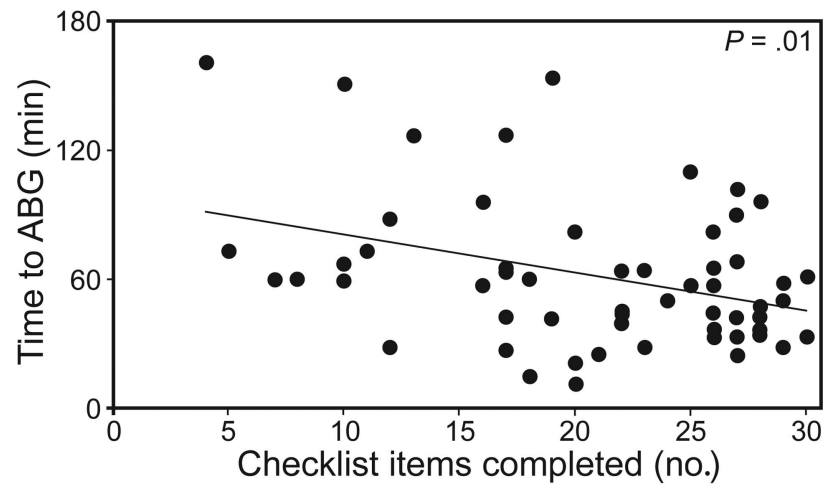

Fig. 3. Time to arterial blood gas (ABG) according to checklist completeness. Checklists were used with 58 subjects in the postintervention period.

need to be clearer to stakeholders. In fact, the checklist title Post-Intubation Time Out tacitly implies applicability to witnessed intubations only. The tool has now been renamed the Mechanical Ventilation Time Out to emphasize the importance of applying the intervention to all mechanically ventilated patients, and this point is stressed during education sessions.

Even with multifaceted educational sessions and targeted emails, altering the behavior of the large number of revolving stakeholders was difficult. Adherence to a complex checklist, which required 3 providers to perform multiple tasks at discrete times, suffered without a defined process leader. The role of process owner should be fulfilled by a team member who is constantly present and may vary with local staffing considerations. Daytime and nighttime process owners should be identified. Unlike the physicians, the vast majority of nurses and respiratory therapists staff our MICU continuously. We can capitalize upon this consistent presence by empowering bedside nurses and respiratory therapists to become checklist process owners, which might improve checklist fidelity.

Prompting and providing performance feedback are additional strategies known to improve checklist adherence and sustainability. ${ }^{15,26} \mathrm{We}$ encourage our personnel to set mobile telephone alarms as deadline reminders during the time-out huddle. An alarm at 15 min prompts ABG collection, and a second alarm at 60 min prompts checklist completion. However, we struggled to provide real-time performance feedback, which may have contributed to the persistently low adherence observed for certain subgroups. We intend to provide feedback in the form of a publicly displayed chart of biweekly outcomes similar to Figure 4 . Additionally, robust monitoring is required to sustain performance, especially in settings with high stakeholder turnover that may impede educational initiatives. Statistical process control using control charts to monitor rates of timely ABGs and checklist completion can detect declining performance below a defined threshold. ${ }^{35}$ These performance decrements should trigger immediate review to identify root causes and design additional sustainability measures.

The main strengths of our study derive from the intervention's design as a tool to both enhance communication (the time out) and reduce variations in care (the checklist), which are 2 leading patient safety strategies. ${ }^{36,37}$ The PostIntubation Time Out employs multiple tactics to reduce variation in $\mathrm{ABG}$ collection. The process is standardized 


\section{Post-Intubation Checklist and Time Out to Expedite Mechanical Ventilation Monitoring}

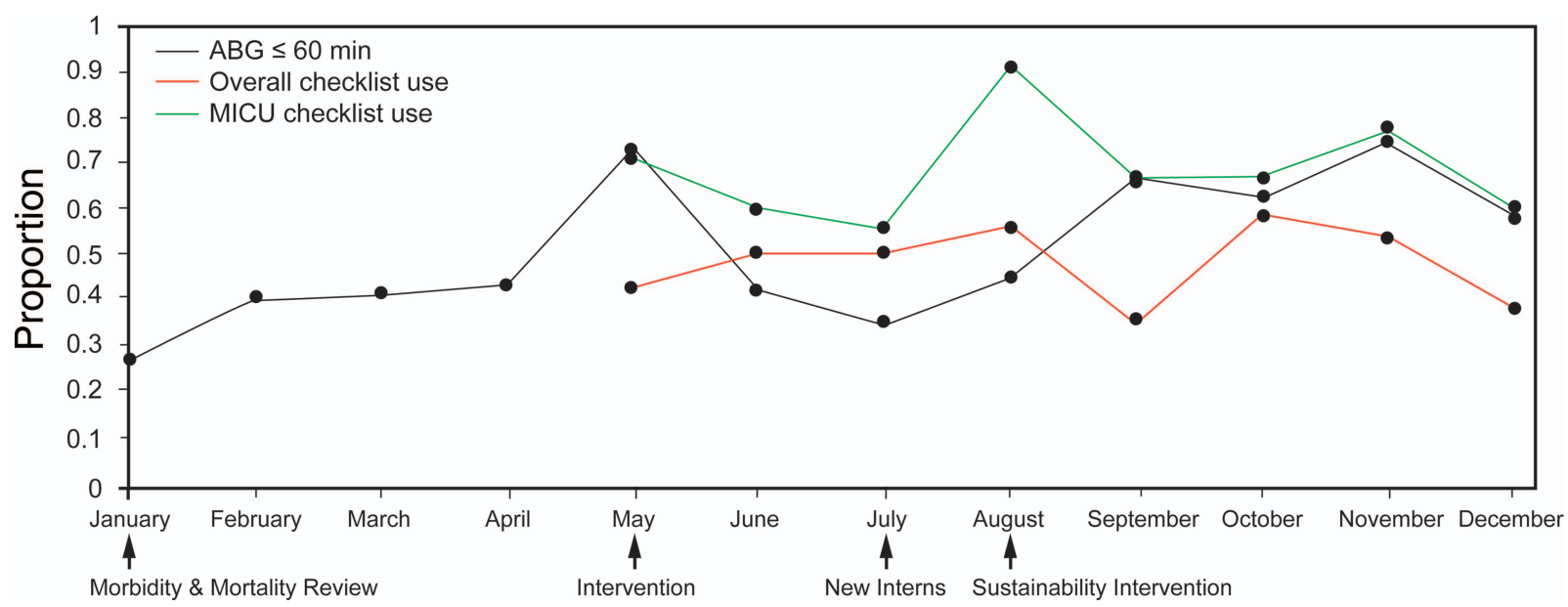

Fig. 4. Post-Intubation Time Out checklist sustainability. The proportion of subjects achieving the primary outcome (arterial blood gas [ABG] results $\leq 60 \mathrm{~min}$ ) and the checklist initiation rates were tracked during each month of the study.

and establishes a guideline for expected ABG timing. Responsibility for ABG collection is assigned to a specific individual of the team's choosing, thereby reducing role ambiguity while preserving flexibility in the face of competing clinical priorities in the early stages of mechanical ventilation. Team members also document completion of assigned tasks, assuring accountability and knowledge sharing in a manner akin to closed-loop communication. ${ }^{38}$ The multidisciplinary manner in which all stakeholders participated in the intervention's construction, pilot testing, and revision solidified the project's legitimacy. Soliciting and incorporating stakeholder feedback probably enhanced the checklist's usability. ${ }^{13}$ Notably, the intervention's strengths are not exclusive to $\mathrm{ABG}$ monitoring and would apply equally to expedite venous blood gas monitoring.

The intervention was designed to facilitate early detection of insufficient ventilation rather than to reduce the frequency of acidemia. The high prevalence of respiratory acidosis and moderate-severe acidemia demonstrates the difficulty in estimating a patient's ventilation requirements and underscores the importance of early monitoring. We have revamped the time out process and revised our checklist to provide decision support and enhance communication about ventilator settings (see the supplementary materials at http://www.rcjournal.com), which may mitigate acidemia. To foster active communication and teamwork, ${ }^{16}$ the bedside nurse will now read the checklist aloud and challenge each team member to verify timely completion of his or her respective tasks. This communication strategy transforms the process from 3 individuals working independently on their own static parallel checklists to a unified static sequential with confirmation and verification checklist. ${ }^{13}$ Revised checklist tasks are now sequenced chronologically to match workflow and are compartmentalized into intubation, immediate post-intubation, and follow-up periods.
We acknowledge multiple study limitations, beginning with the uncontrolled before-and-after observational study design that is often employed in quality improvement studies. It was not feasible to randomize subjects to receive the intervention due to confounding from the MICU team structure, with nurses and respiratory therapists integrated across both physician teams. A difference-in-differences analysis comparing $\mathrm{ABG}$ timing with a control ICU that did not receive the intervention would account for secular performance trends; such a design was not possible because the house staff and some respiratory therapists exposed to the intervention also rotate through the other ICUs in our hospital. To mitigate the influence of unmeasured secular trends, we selected the pre-intervention time period to begin after morbidity and mortality review heightened health system awareness of ventilation monitoring. Second, the lack of blinding introduced a risk of bias. Data were collected by a single non-blinded researcher; however, subjects were randomized, a structured data collection form was used to collect objective data, and analyses were performed by an independent statistician. The Hawthorne effect ${ }^{39}$ was probably minimized by applying the Post-Intubation Time Out to all patients as the new standard of care. Third, the primary outcome of the quality improvement initiative was time to ABG sampling, the significance of which is unclear in relation to more clinically meaningful outcomes, such as duration of mechanical ventilation or mortality. Fourth, the timeline for obtaining an ABG result can also be shortened by targeting the ABG specimenprocessing pathway, which our study did not address. Although most delays occur during ABG collection rather than during laboratory processing (data not shown), installing point-of-care blood gas analyzers would eliminate the time required to transport the specimen and enter test results in the electronic health record. Finally, ABG timing is only one facet of the checklist and time out process. We 


\section{Post-Intubation Checklist and Time Out to Expedite Mechanical Ventilation Monitoring}

suspect that there is value in prompting the many other important post-intubation tasks, such as lung mechanics assessment, lung-protective ventilation in acute lung injury, and sputum culture collection, although we recognize that this was beyond the scope of the current study.

\section{Conclusions}

Prompt detection of inadequate ventilation is important due to the adverse consequences of severe acidemia and the inherent difficulty in accurately estimating a patient's initial ventilation needs. We developed a multidisciplinary time-out process and checklist to coordinate the multiple tasks that should occur immediately post-intubation, including enhanced early monitoring of mechanical ventilation via expedited $\mathrm{ABG}$ assessment. Checklist adherence was associated with faster ABGs in a dose-dependent manner, although checklist underutilization among certain patient subgroups underscored opportunities for future process revisions. Sustained performance improvement required several months to achieve, reflecting a possible learning curve associated with behavioral change. Key tenets for effectively implementing a change in ICU workflow include: articulating a compelling rationale to motivate stakeholders, incorporating stakeholder input into workflow redesign, educating all participants, delegating a consistent process leader (especially in settings with high stakeholder turnover), monitoring performance, providing timely feedback, and reinforcing behavioral change.

\section{ACKNOWLEDGMENTS}

We acknowledge the important contributions of colleagues at the University of Pennsylvania. Dr Anjali Gopalan and Dr Joanna Hart focused early attention on the problem of delayed mechanical ventilation monitoring. Cheryl Maguire and Jessica Fuller provided leadership and nursing education. Dr Jennifer Myers and Dr Neha Patel continue to inspire and facilitate trainee involvement in quality improvement. Statistician Xingmei Wang offered exceptional statistical support.

\section{REFERENCES}

1. Metnitz PG, Metnitz B, Moreno RP, Bauer P, Del Sorbo L, Hoermann $\mathrm{C}$, et al. Epidemiology of mechanical ventilation: analysis of the SAPS 3 database. Intensive Care Med 2009;35(5):816-825.

2. Wunsch H, Linde-Zwirble WT, Angus DC, Hartman ME, Milbrandt EB, Kahn JM. The epidemiology of mechanical ventilation use in the United States. Crit Care Med 2010;38(10):1947-1953.

3. Jung B, Rimmele T, Le Goff C, Chanques G, Corne P, Jonquet O, et al. Severe metabolic or mixed acidemia on intensive care unit admission: incidence, prognosis and administration of buffer therapy: a prospective multiple-center study. Crit Care 2011;15(5):R238.

4. Shirani F, Salehi R, Naini AE, Azizkhani R, Gholamrezaei A. The effects of hypotension on differences between the results of simultaneous venous and arterial blood gas analysis. J Res Med Sci 2011; 16(2):188-194.

5. Williams KB, Christmas AB, Heniford BT, Sing RF, Messick J. Arterial vs venous blood gas differences during hemorrhagic shock. World J Crit Care Med 2014;3(2):55-60.
6. Adrogué HJ, Rashad MN, Gorin AB, Yacoub J, Madias NE. Assessing acid-base status in circulatory failure. Differences between arterial and central venous blood. N Engl J Med 1989;320(20):13121316.

7. Buehler S, Jensen MC, Lozano-Zahonero S, Gottlieb D, Eckle D, Szczyrba M, et al. The dynamics of carbon dioxide equilibration after alterations in the respiratory rate. Physiol Meas 2013;34(9): 1151-1161.

8. Taskar V, John J, Larsson A, Wetterberg T, Jonson B. Dynamics of carbon dioxide elimination following ventilator resetting. Chest 1995; 108(1):196-202.

9. Solis R, Anselmi C, Lavietes M, Khan MA. Rate of decay or increment of $\mathrm{PaO}_{2}$ following a change in supplemental oxygen in mechanically ventilated patients with diffuse pneumonia. Chest 1993; 103(2):554-556.

10. Cakar N, Tuŏrul M, Demirarslan A, Nahum A, Adams A, Akýncý O, et al. Time required for partial pressure of arterial oxygen equilibration during mechanical ventilation after a step change in fractional inspired oxygen concentration. Intensive Care Med 2001;27(4):655659.

11. Sutcliffe KM, Lewton E, Rosenthal MM. Communication failures: an insidious contributor to medical mishaps. Acad Med 2004;79(2): 186-194.

12. Greenberg CC, Regenbogen SE, Studdert DM, Lipsitz SR, Rogers SO, Zinner MJ, Gawande AA. Patterns of communication breakdowns resulting in injury to surgical patients. J Am Coll Surg 2007; 204(4):533-540.

13. Winters BD, Gurses AP, Lehmann H, Sexton JB, Rampersad CJ, Pronovost PJ. Clinical review: checklists: translating evidence into practice. Crit Care 2009;13(6):210.

14. Haynes AB, Weiser TG, Berry WR, Lipsitz SR, Breizat AH, Dellinger EP, et al. A surgical safety checklist to reduce morbidity and mortality in a global population. N Engl J Med 2009;360(5):491499.

15. Weiss CH, Moazed F, McEvoy CA, Singer BD, Szleifer I, Amaral LA, et al. Prompting physicians to address a daily checklist and process of care and clinical outcomes: a single-site study. Am J Respir Crit Care Med 2011;184(6):680-686.

16. Joint Commission Universal Protocol for Preventing Wrong Site, Wrong Procedure, and Wrong Person Surgery. http://www.jointcommission. org/standards_information/up.aspx. Accessed March 25, 2015.

17. Pronovost P, Needham D, Berenholtz S, Sinopoli D, Chu H, Cosgrove $\mathrm{S}$, et al. An intervention to decrease catheter-related bloodstream infections in the ICU. N Engl J Med 2006;355(26):27252732.

18. Berenholtz SM, Pham JC, Thompson DA, Needham DM, Lubomski LH, Hyzy RC, et al. Collaborative cohort study of an intervention to reduce ventilator-associated pneumonia in the intensive care unit. Infect Control Hosp Epidemiol 2011;32(4):305-314.

19. Arriaga AF, Bader AM, Wong JM, Lipsitz SR, Berry WR, Ziewacz JE, et al. Simulation based trial of surgical-crisis checklists. N Engl J Med 2013;368(3):246-253.

20. Langley GJ, Moen RD, Nolan KM, Nolan TW, Norman CL, Provost LP. The improvement guide: a practical approach to enhancing organizational performance, 2nd edition. New York: Jossey-Bass;2009: 97-99, 175-178.

21. Waydhas C, Schneck G, Duswald KH. Deterioration of respiratory function after intra-hospital transport of critically ill surgical patients. Intensive Care Med 1995;21(10):784-789.

22. Parmentier-Decrucq E, Poissy J, Favory R, Nseir S, Onimus T, Guerry MJ, et al. Adverse events during intrahospital transport of critically ill patients: incidence and risk factors. Ann Intensive Care 2013; 3(1):10. 


\section{Post-Intubation Checklist and Time Out to Expedite Mechanical Ventilation Monitoring}

23. Ligtenberg JJ, Arnold LG, Stienstra Y, van der Werf TS, Meertens JH, Tulleken JE, Zijlstra JG. Quality of interhospital transport of critically ill patients: a prospective audit. Crit Care 2005;9(4):R446-R451.

24. Droogh JM, Smit M, Hut J, de Vos R, Ligtenberg JJ, Zijlstra JG. Inter-hospital transport of critically ill patients: expect surprises. Crit Care 2012;16(1):R26.

25. Knaus WA, Wagner DP, Draper EA, Zimmerman JE, Bergner M, Bastos PG, et al. The APACHE III prognostic system: risk prediction of hospital mortality for critically ill hospitalized adults. Chest 1991; 100(6):1619-1636.

26. Merlani P, Garnerin P, Diby M, Ferring M, Ricou B. Linking guideline to regular feedback to increase appropriate requests for clinical tests: blood gas analysis in intensive care. BMJ 2001;323(7313):620624

27. Harrison RV, Payne BC. Developing criteria for ordering common ancillary services. Med Care 1991;29(9):853-877.

28. Martin DS, Grocott MP. Oxygen therapy in critical illness: precise control of arterial oxygenation and permissive hypoxemia. Crit Care Med 2013;41(2):423-432.

29. Albert MS, Dell RB, Winters RW. Quantitative displacement of acid-base equilibrium in metabolic acidosis. Ann Intern Med 1967; 66(2):312-322.

30. Neily J, Mills PD, Young-Xu Y, Carney BT, West P, Berger DH, et al. Association between implementation of a medical team training program and surgical mortality. JAMA 2010;304(15):1693-1700.

31. Pickering SP, Robertson ER, Griffin D, Hadi M, Morgan LJ, Catchpole $\mathrm{KC}$, et al. Compliance and use of the World Health Organization checklist in U.K. operating theatres. Br J Surg 2013;100(12): 1664-1670.
32. van Klei WA, Hoff RG, van Aarnhem EE, Simmermacher RK, Regli LP, Kappen TH, et al. Effects of the introduction of the WHO "Surgical Safety Checklist" on in-hospital mortality: a cohort study. Ann Surg 2012;255(1):44-49.

33. Levy SM, Senter CE, Hawkins RB, Zhao JY, Doody K, Kao LS, et al. Implementing a surgical checklist: more than checking a box. Surgery 2012;152(3):331-336.

34. Cullati S, Le Du S, Raë AC, Micallef M, Khabiri E, Ourahmoune A, et al. Is the Surgical Safety Checklist successfully conducted? An observational study of social interactions in the operating rooms of a tertiary hospital. BMJ Qual Saf 2013;22(8):639-646.

35. Thor J, Lundberg J, Ask J, Olsson J, Carli C, Härenstam KP, Brommels M. Application of statistical process control in healthcare improvement: systematic review. Qual Saf Health Care 2007;16(5): 387-399.

36. Leonard M, Graham S, Bonacum D. The human factor: the critical importance of effective teamwork and communication in providing safe care. Qual Saf Health Care 2004;13(Suppl 1):i85-i90.

37. Leotsakos A, Zheng H, Croteau R, Loeb JM, Sherman H, Hoffman $\mathrm{C}$, et al. Standardization in patient safety: the WHO High 5 s project. Int J Qual Health Care 2014;26(2):109-116.

38. Burke CS, Salas E, Wilson-Donnelly K, Priest H. How to turn a team of experts into an expert medical team: guidance from the aviation and military communities. Qual Saf Health Care 2004;13(Suppl 1): i96-i104.

39. McCarney R, Warner J, Iliffe S, van Haselen R, Griffin M, Fisher P. The Hawthorne Effect: a randomised, controlled trial. BMC Med Res Methodol 2007;7:30.

This article is approved for Continuing Respiratory Care Education credit. For information and to obtain your CRCE

(free to AARC members) visit

www.rcjournal.com

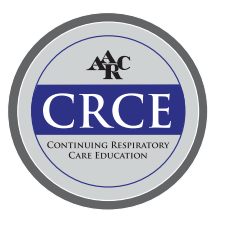

